\title{
O programa Aedes em Foco como elemento de combate a arboviroses
}

\author{
André J. Monteiro ${ }^{1}$, Caio G. C. S. Cavalcanti ${ }^{2}$, Ernesto T. de Lima, ${ }^{1}$ Joaquim F. C. Neto ${ }^{1}$, \\ Ivo C. B. Coelho ${ }^{1}$, Gabriel A. L. Paillard ${ }^{1}$, George A. M. Gomes ${ }^{1}$, Francisco G. G. Junior ${ }^{1}$, \\ Henrique S. L. Pequeno ${ }^{1}$, Miguel F. de Castro ${ }^{1}$, Rafael A. F. do Carmo ${ }^{1}$, Rubson P. Maia ${ }^{1}$

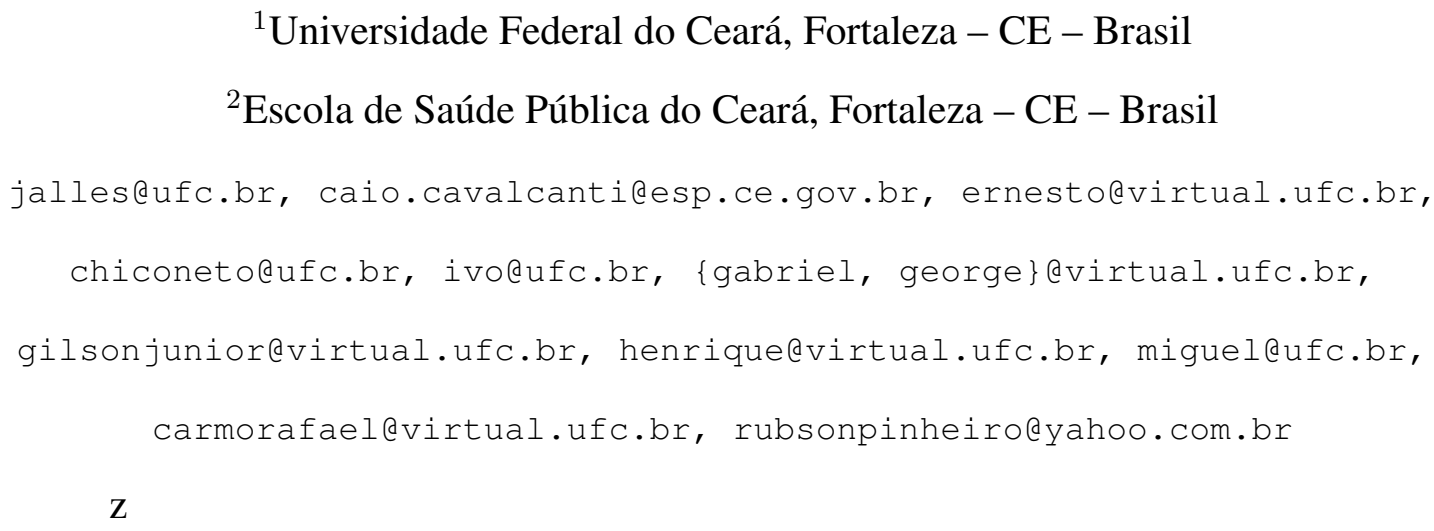

\begin{abstract}
This paper presents the application of the Aedes em Foco project to the combat of arbovirosis, especially those caused by the Aedes Aegypti mosquito. It presents the current state of combat to this problem in Brazil, the model proposed and the results obtained in a a study case. The case study has been performed at the city of Cedro - Ceará and the project contributed to changes in the way mandatory residential visits are performed. Through the software platform and auxiliary elements introduced by the project, the observed results empirically demonstrate that the project contributed to a better understanding and control of the arbovirus control process in this municipality.
\end{abstract}

Resumo. Este artigo apresenta a aplicação do projeto Aedes em Foco no combate à arboviroses, principalmente as causadas pelo mosquito Aedes Aegypti. Apresenta o estado atual de combate a esse problema no Brasil, o modelo proposto pelo projeto e os resultados obtidos em um estudo de caso. O estudo de caso foi realizado na cidade de Cedro - Ceará e o projeto contribuiu para mudanças na maneira como são realizadas visitas residenciais obrigatórias por Agentes de Endemias. Por meio da plataforma de software e dos elementos auxiliares introduzidos pelo projeto, os resultados observados demonstram empiricamente que o projeto contribuiu para uma melhor compreensão e controle do processo de combate ao arbovírus nesse município.

\section{Introdução}

Arboviroses são doenças causadas por arbovírus cuja ocorrência acontece prioritariamente em áreas silvestres. Porém, o crescimento de epidemias urbanas como da Dengue, Mayaro e Chikungunya é crescente e notável. [Rosa et al. 2000].

Há muito a população brasileira tem sido vítima de arboviroses, o que tem trazido inúmeros prejuízos ao país. O controle sanitário ocorre em diversas frentes, como, por 
exemplo, pesquisas para criação de vacinas [Bricks 2004], manipulação genética de vetores para controle biológico e desenvolvimento de novos larvicidas [Oliveira et al. 2017]. Até o presente momento, as políticas públicas de enfrentamento pouco tem avançado. [Valle et al. 2016] A cada novo ano, há recorrência e aumento no registro de caso, além da diversificação da capacidade de infecção pelos vetores. Um outro importante aspecto que caracteriza o combate às arboviroses no Brasil é a carência de estratégias articuladas entre os diversos órgãos públicos, organizações sociais e profissionais de atuação direta [Pessoa et al. 2013, Silva et al. 2015b].

É perceptível que os atores governamentais brasileiros ligados à saúde pública estão solitários na responsabilização pelo trabalho de prevenção, monitoramento, eliminação e controle de vetores causadores de arboviroses, mais precisamente, o mosquito Aedes aegypti. Como alerta [Araujo 2012],"A dengue, como todos os demais agravos da saúde, é um fenômeno multidimensional e como tal exige que seja considerado de forma multidisciplinar e multissetorial."A atuação desacompanhada reduz novas propostas para erradicar as arboviroses. Além de não contar com possíveis benefícios inerentes ao trabalho articulado de múltiplas competências, perde-se uma chance da analise integrada de dados, com cobertura e correlação maior de variáveis. Nota-se, ainda, oportunidades de uso das Tecnologias Digitais da Comunicação e Informação (TDICs), especialmente na tipologia informacional, informatização dos processos, acessibilidade de dados e visualização das informações coletadas.

Esta pesquisa-ação apresenta o Programa Aedes em Foco como uma nova abordagem para gestores públicos, profissionais de saúde e cidadãos de atuação na captação de informações, análise de dados e atuação social de controle das arboviroses transmitidas pelo mosquito Aedes aegypti. A abordagem proposta se concentra em aspectos de obtenção, integração, análise, sistematização e apresentação de dados, permitindo que diversos especialistas (epidemiologistas, bioestatísticos etc) elaborem suas análises com mais precisão (refinamento de dados) e agilidade (tempo real), através da construção de um app móvel, um sistema web, um jogo digital, uma campanha de mobilização social e cursos online.

A pesquisa-ação [Santos et al. 2018] aqui apresentada, foi aplicada no município de Cedro, localizado no interior cearense, durante os anos de 2018 e 2019. Ele demonstra que os recursos disponibilizados permitem uma tomada de decisões mais eficiente e imediata por parte de gestores públicos, ampliando a otimização de uso de recursos e a eficácia dos esforços das equipes de trabalho vinculadas à causa.

Desta forma, o Aedes em Foco se define como uma plataforma de e-Health [Eysenbach 2001] com foco na coordenação, controle e melhoria do processo de combate às arboviroses, especialmente aos males vinculados ao mosquito Aedes aegypti, através do envolvimento de diversos atores sociais e da análise dos dados gerados pela atuação colaborativa, através da tecnologia sanitária, integração tecnológica, social e educacional [Schall et al. 2015, Quintão 2016, Neto 2016] ofertadas pelo Programa.

\subsection{Cenário atual de combate a arboviroses}

Atualmente, as ações de enfrentamento às arboviroses têm como elementos centrais dois grupos de servidores municipais: Agentes de Combate a Endemias (ACE) e Agentes Comunitários de Saúde (ACS) [Pessoa et al. 2016]. Dentro da Estratégia de Saúde da 
Família $^{1}$, do Ministério da Saúde, ACE são funcionários municipais responsáveis pela visita a residências, estabelecimentos comerciais e terrenos desocupados para ações de prevenção e controle de doenças. Já ACS são profissionais que trabalham em comunidades específicas - normalmente perto de suas moradias - em ações de orientação sanitária [Nunes et al. 2002]. A familiaridade espacial é importante para que os agentes adicionem ao seu trabalho o conhecimento e a confiança das pessoas atendidas [Pessoa et al. 2016]. Os agentes nutrem diferentes sistemas governamentais de informação que são utilizados como recursos-chave para o monitoramento de arboviroses. No entanto, muitas das tecnologias em uso apresentam inconsistência e atraso no provimento e disponibilização informacional.

Existem vários aplicativos disponíveis gratuitamente vinculados à causa ${ }^{2}$. Os exemplos trazem, recorrentemente, a realização de denúncias de focos para os órgãos públicos responsáveis pelo controle sanitário como função principal. Outros softwares auxiliam no processo verificação semanal individual e oferta de informações sobre o mosquito e as formas de prevenção das arboviroses.

Partindo, inicialmente, de uma proposta similar aos aplicativos já disponíveis, os integrantes do Aedes em Foco compreenderam que nenhuma oferta promovia significativa alteração na natureza dos dados ou no fluxo informacional e mecanismos de combate às doenças provocadas pelo Aedes aegypti. Com o avanço da pequisa-ação, os softwares tornaram-se meio auxiliar para propor inovações nas tradicionais operações relacionadas ao controle do mosquito.

Com o advento do e-SUS ${ }^{3}$ [Oliveira et al. 2016], alguns aplicativos para dispositivos móveis, como o e-SUS AB Território, buscaram facilitar o processo de submissão de dados coletados - principalmente por ACS - durante visitas domiciliares. Entretanto, o processo de visita domiciliar realizado pelos Agentes não sofreu ganhos significativos nas últimas décadas. Durante as visitas dos ACEs, há a busca por possíveis locais de reprodução de mosquitos, aplicação de larvicidas, eliminação de focos e registro escrito da inspeção em formulários impressos. Posteriormente, os conteúdos são digitalizados. $\mathrm{O}$ processo de transcrição pode levar até 2 semanas, segundo os responsáveis pelo processo entrevistados durante a pesquisa-ação. $\mathrm{O}$ atraso quinzenal equivale, coincidentemente, ao ciclo de vida do mosquito [Junior et al. 2013].

Outros estudos [Duarte and França 2006, Feitosa et al. 2016] reportam um hiato de, em média, 15 dias na transferência dos dados para o ambiente digital. Só então, as informações coletados podem ser acessadas e, se desejável, utilizadas. É importante apontar que, até junho de 2020, apenas os dados coletados pelos ACS são disponibilizados na plataforma e-SUS.A pesquisa-ação identificou que o tempo necessário para a disponibilização dos dados pode dificultar a eficácia de uso das informações. E um dos primeiros obstáculos para o rápido fluxo dos dados é o esforço empregado entre o registro manual, transferência de arquivos, digitação e inclusão na plataforma digital. A jornada dos dados pode retardar o acesso, a integração e a análise dos conteúdos coletados.

\footnotetext{
${ }^{1}$ http://www.saude.gov.br/acoes-e-programas/saude-da-familia/agente-comunitario-de-saude

${ }^{2}$ http://multirio.rio.rj.gov.br/index.php/leia/reportagens-artigos/reportagens/12086-cresce-número-deaplicativos-de-combate-ao-i-aedes-aegypti-i

${ }^{3}$ http://esus.saude.ms.gov.br/
} 


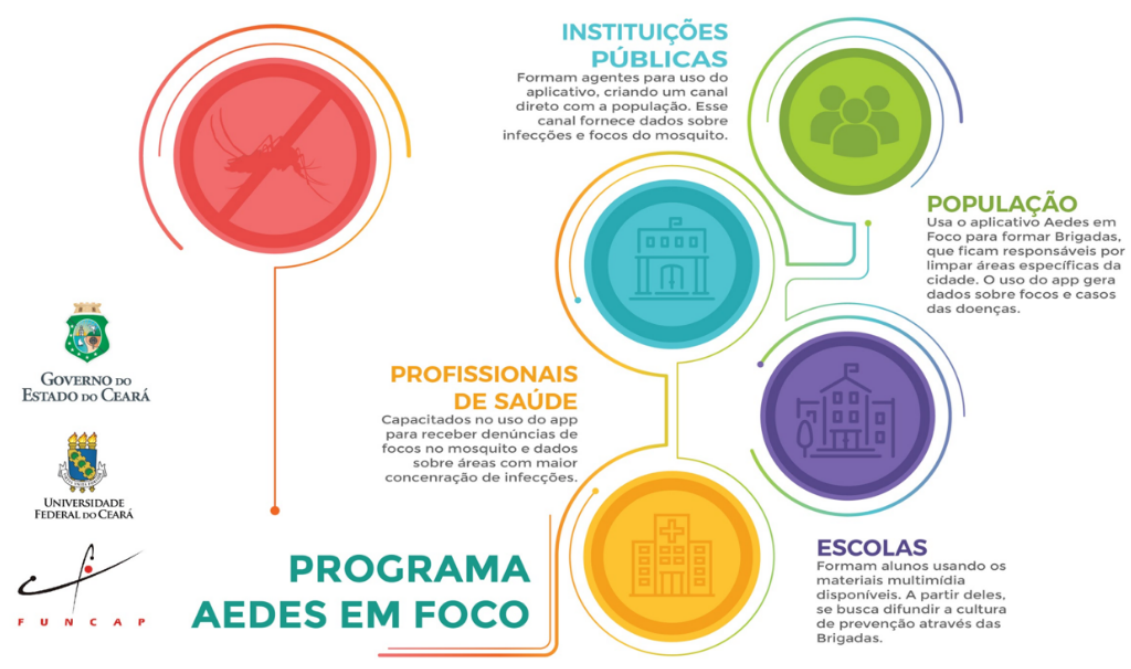

Figura 1. Públicos estratégicos do Programa Aedes em Foco

Tomando como exemplo o processo de coleta de dados empreendido pelo Sistema de Informação de Agravos de Notificação (SINAN), em períodos epidêmicos - pelo grande volume de formulários impressos utilizados durante as inspeções - o processo de digitalização para o sistema pode demorar meses, quando o cenário analisado é um grande centro urbano. Precisamente, quando o rápido acesso aos dados para tomada de decisão torna-se ainda mais valioso, aumenta a demora para a disponibilização de informações.

O segundo provável obstáculo consiste na dificuldade de identificação qualificada dos dados. O preenchimento manual ocasiona, de forma recorrente, a ilegibilidade de informações, incompletude de dados e imprecisão na identificação dos locais inspecionados. Finalmente, um terceiro obstáculo observado consiste na desmobilização dos profissionais à causa. Não é possível visualizar a relevância dos dados coletados em cada inspeção e do somatório dos esforços laborais. Da mesma forma, não há uma notabilização do impacto do trabalho individual e coletivo realizado pelos ACS e ACEs.

\section{Metodologia}

A pesquisa-ação identificou que os processos de modernização das estratégias de combate às arboviroses, atualmente, ocorrem sem a participação ativa dos profissionais quem atuam diariamente e diretamente no controle municipal das doenças: ACSs, ACEs e integrantes das secretarias de saúde municipais.

A abordagem orientada à inovação do Aedes em Foco aproximou a equipe de desenvolvedores do Programa e os profissionais de saúde de Cedro. Juntos, a equipe científica, os ACS e os ACEs dialogaram sobre as diferentes arboviroses, discutiram o fluxo de coleta de dados e vivenciaram as rotinas de visitas. Com a pesquisa-ação, o protótipo do software sofreu alterações com a revisão, inclusão e exclusão de funcionalidades orientadas ao propósito de dar rápido acesso aos dados que proporcionem maior eficácia na análise de cenários e tomada de decisões vinculadas às políticas e ações de saúde pública. Assim, a pesquisa-ação do Programa Aedes em Foco cumpriu os seguintes objetivos:

1. Criação colaborativa de um aplicativo mobile para uso em visitas domiciliares ligadas ao controle de arboviroses; 


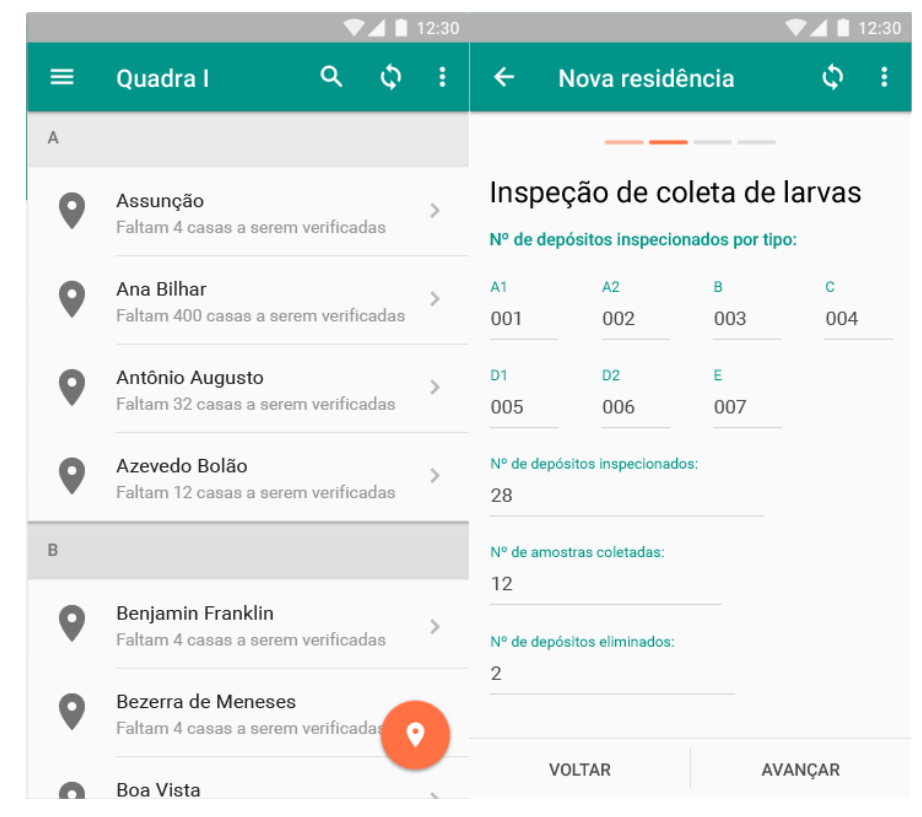

Figura 2. Telas do aplicativo Aedes em Foco - ACE

2. Desenvolvimento uma infraestrutura tecnológica para a sistematização e otimização da coleta e análise de dados em tempo real;

3. Mobilização de ACS, ACE, Gestores de Saúde Pública e comunidade para a compreensão da causa e participação efetiva no combate às arboviroses.

A Figura 1 ilustra o propósito de integração tecnológica, social e educacional do programa Aedes em Foco direcionado, primeiramente, aos profissionais de saúde que trabalham em campo e adicionando os gestores e funcionários de saúde municipal vinculados à causa, além de integrantes da rede de ensino pública e instituições de grande capilaridade social como igrejas e veículos de comunicação.

\subsection{Desenvolvimento Tecnológico}

O primeiro artefato do Programa desenvolvido foi aplicativo móvel Aedes em Foco Agente de Combate a Endemias. Ele permite a automatização do processo de controle e registro de verificações em imóveis feitas por ACEs. Tal documentação faz parte das responsabilidades dos ACEs, segundo as "Diretrizes Nacionais para a Prevenção e Controle de Epidemias de Dengue" [Brasil 2009, p. 70] e o "Programa Nacional de Controle da Dengue - PNCD" [Brasil 2002]. O formulário traz informações do endereço visitado, locais de acúmulo de de água inspecionados e - quando adequado - eliminados, além de amostras de larvas coletadas e tipo de intervenção sanitária aplicada.

O aplicativo foi desenvolvido para o sistema operacional Android e disponibilizado gratuitamente na Google Play Store. Seus requisitos, restrições e arquitetura informacional foram definidos após entrevistas e diálogos com profissionais da Prefeitura Municipal de Cedro, ACEs, somados à análise dos documentos mandatórios requisitados pelo Ministério da Saúde e contando, ainda, com as observações de profissionais da Escola de Saúde Pública da Faculdade de Medicina da Universidade Federal do Ceará.

A Figura 2 apresenta dois screenshots do aplicativo referentes ao preenchimento de endereços visitados e locais de acúmulo de água inspecionados e - quando adequado 


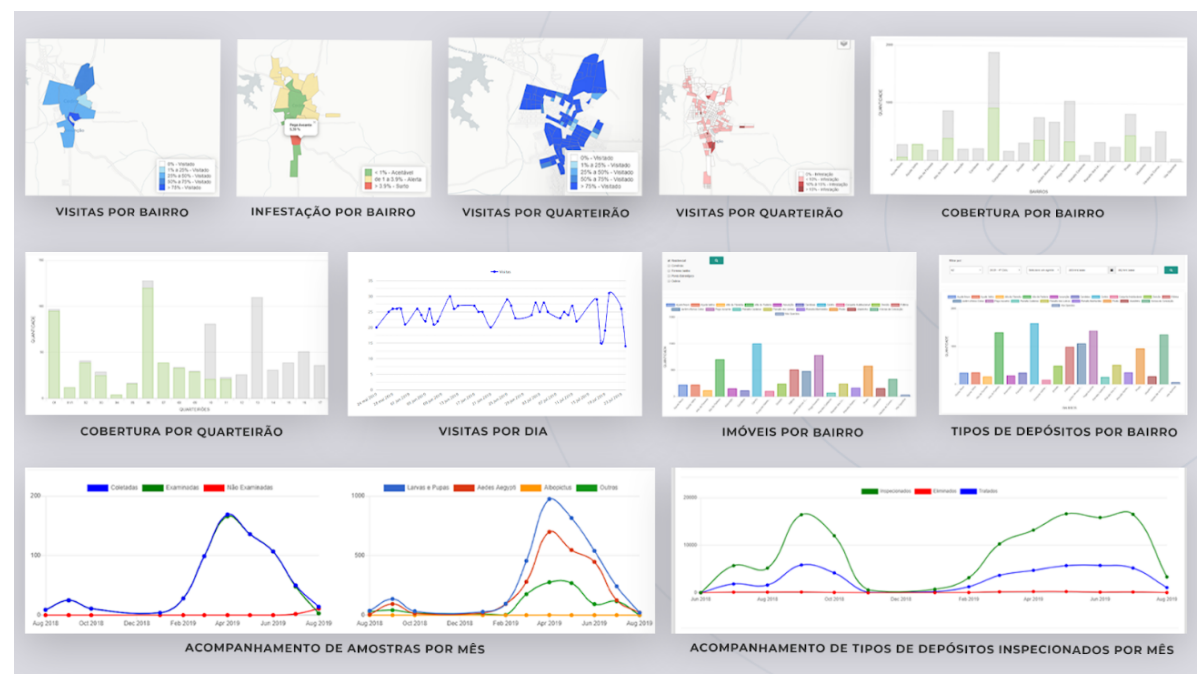

Figura 3. Dashboard de sumarização de dados do Aedes em Foco

- eliminados. Os dados das verificações são transferidos online para o banco de dados integrado do Aedes em Foco quando há acesso à internet disponível. As informações são aglutinadas e apresentadas no painel de controle do Aedes em Foco, tal como mostrado na Figura 3. O dashboard disponibiliza vários indicadores referentes às inspirações presenciais. Elas podem ser organizadas em bairros, quarteirões, tipos de locais de acúmulo de água, linha temporal e conteúdos referentes ao LIRAa [Brasil 2012]. Merece destaque o envio imediado e automático de dados para o sistema do e-SUS.

Para os gestores das equipe de ACEs, o sistema de gerenciamento do Aedes em Foco também auxilia o processo de organização tática de atividades em campo. O mapa com todos os imóveis da município é armazenado no sistema e os funcionários da secretaria municipal de saúde podem visualizar regiões, avaliar dados de áreas específicas e definir quais quarteirões serão visitados por cada agente. Também é possível automatizar o controle dos chamados ciclos: período em que determinado número de visitas é feito. Após a conclusão das inspirações em todo perímetro elencado, o ciclo é encerrado e um novo ciclo é iniciado.

\subsection{Mobilização social e educação}

Estratégias de educação e mobilização são elementos importantes [Silva et al. 2015b] nas políticas públicas de combate às arboviroses. Elas tem a missão de informar e conclamar a população para a participação colaborativa nas ações sanitárias. O programa Aedes em Foco integrou atividades de formação, capacitação e comunicação comunitária, empreendendo formações para diferentes públicos estratégicos, como: ACS, ACE, professores do ensino fundamental e médio, estudantes, funcionários públicos de saúde e formadores de opinião (radialistas, lideranças comunitárias e integrantes de movimentos sociais) através dos formatos presencial e semipresencial, fazendo uso do ambiente virtual de aprendizagem Solar [Coutinho et al. 2013] e abordando aspectos sobre arboviroses e estratégias de controle. Assim, foram propostos e desenvolvidos os seguintes cursos:

- Educação em ação contra o Aedes Aegypti;

- Curso básico de formação de brigadas de combate ao Aedes aegypti; 


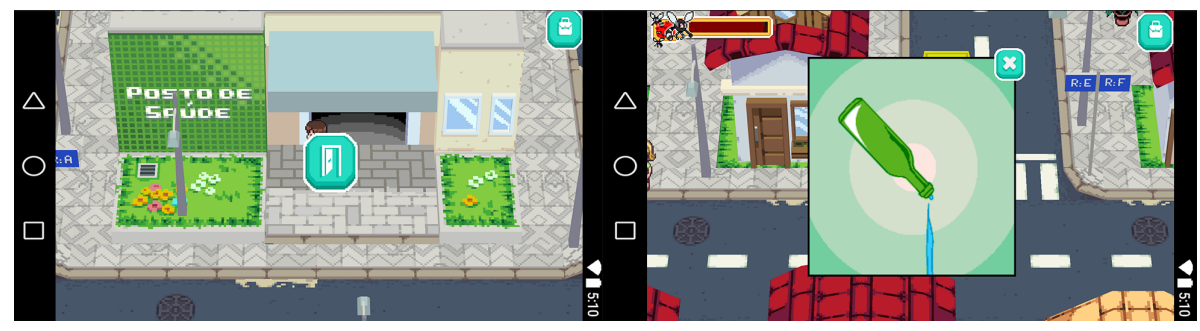

Figura 4. O jogo Aedes em foco

- Programação e Diversão: Desenvolvendo o raciocínio lógico-matemático com programação de forma lúdica;

- Eu e os robôs: criando e recriando seres artificiais;

- Criar, programar e Jogar: criando jogos digitais;

- Luz, câmera e criação: como fazer vídeos digitais para redes sociais e jogos.

Os primeiros dois cursos abordaram conhecimentos gerais para a eliminação de focos do mosquito. Os três seguintes trataram do ensino lúdico de conceitos de programação e automação através da plataforma Arduíno [McRoberts 2015] voltados ao estímulo criativo de geração de soluções tecnológicas inovadoras para o combate às arboviroses. A última ação foi destinada à melhoria da comunicação em redes sociais digitais.

De forma acessória, também foi desenvolvido o jogo Aedes em Foco, disponível na Google Play Store e com screenshots apresentados na Figura 4. Ele consiste em um jogo digital no qual o participante explora uma cidade infestada de mosquitos portadores de arbovírus. Para salvar o local, é preciso efetuar diversas tarefas de combate como a criação de brigadas locais. Este instrumento de ação social colaborativa conta com o aplicativo Aedes em Foco - Brigadas como ferramenta para a formação e gestão de grupos de pessoas que, com o apoio do software, podem registrar e eliminar pontos de infestação, além de monitorar periodicamente as atividades de inspeção.

\section{Pesquisa-Ação}

Durante anos de 2018 e 2019 deu-se a condução do projeto piloto no município de Cedro, no estado do Ceará. A escolha do município se deu pelo interesse do gestor municipal em sediar o projeto-piloto e dar suporte suficiente para o trabalho. Desta forma, os diversos elementos do Programa Aedes em Foco foram realizados em diferentes momentos. Nesta seção discutiremos as ações empreendidas e os resultados observados.

Procedimento e Resultados: Visitas in loco foram realizadas pela equipe técnicocientífica com periodicidade mensal - por vezes, quinzenal - ao município de Cedro. As primeiras viagens tinham como propósito a apresentação do projeto, a mobilização dos públicos estratégicos e a compreensão do cenário para a modelagem de táticas e artefatos.

Inicialmente, houve boa aceitação da utilização dos aplicativos, principalmente o Aedes em Foco - ACE pelos gestores da secretaria de saúde municipal. Porém, os 19 agentes não demonstraram interesse em aprender e utilizar o aplicativo. Foi relatado, pelas lideranças dos ACEs, em diversos momentos, o temor dos agentes. Eles acreditavam que o aplicativo seria usado como mecanismo de controle, informando aos supervisores todas as ações dos agentes em campo como, por exemplo, o tempo gasto em cada visita, 
a localização em tempo real e o período de início e finalização do expediente laboral. Também foi relatada uma resistência em abandonar o uso das folhas impressas.

Entretanto, o aplicativo Aedes em Foco - ACE não tem como finalidade o monitoramento dos profissionais, mas sim a otimização do fluxo de dados, visando a eficácia no combate às arboviroses e a promoção da saúde coletiva. As ações de capacitação (descritas a seguir) cessaram a resistência. Ao final do Projeto, 100\% dos ACEs aderiram ao aplicativo e são unânimes em sua escolha se comparado ao processo anterior. Os dashboards de monitoramento do Aedes em Foco permitem a visualização e análise amigável dos dados das visitas em nível de quarteirões. A partir da avaliação visual, foram identificadas regiões já visitadas onde havia grande incidência de focos do Aedes aegypti. Com isso, surgiu a ideia de realizar um mutirão, contando com os agentes municipais, a equipe científica e técnica do Programa, além de cidadãos cedrenses, nos quarteirões com maiores registros de focos.

A 5 mostra a sequência de três ciclos utilizando o Aedes em Foco. O Programa permitiu ao gestores e aos profissionais de saúde identificar as áreas com maior incidência de fatores de risco (em vermelho). Com isso, os gestores sanitários organizaram e realizaram mutirões nos locais específicos com mais registros de infestação. A amostra pontual aponta uma melhoria significativa do nível de infestações observados na cidade de Cedro. Os gestores sanitários entrevistados expressaram satisfação pela possibilidade de intervenção assertiva e otimização de recursos possibilitadas pelo Aedes em Foco. Eles reportaram a fácil visualização de dados que possibilitou uma rápida e inteligente tomada de decisões.

Os cursos oferecidos pelo Aedes em Foco tiveram a participação de mais de uma centena de estudantes, professores, servidores e cidadãos cedrenses. Os participantes originaram as primeiras brigadas voluntárias com o uso do aplicativo Aedes em Foco Brigadas. Inicialmente, os grupos foram cadastrados, houve o registro de atividades no aplicativo e inicio do trabalho voluntário de visita e educação comunitária.

Com o tempo, verificou-se uma gradual dissolução do tamanho e atividade das brigadas. A hipótese inicial que a percepção de participação individual e exercício coletivo seriam suficientes para a manutenção e crescimento da adesão. Porém, em Cedro, as brigadas voluntárias findaram. Acreditamos que estudos metodológicos acerca de motivação em trabalho voluntário e comunitário [Cavalcante 2013] podem guiar a criação de novas táticas e recursos que promovam o sucesso destas brigadas voluntárias.

Por fim, a pesquisa-ação gerou ações não previstas inicialmente pelo projeto. Elas responderam observações, interpretações, questionamentos e aprendizados durante a aplicação do Programa. A apresentação das atividades e resultados seminais merece citação, pois eles integraram efetivamente o projeto, mas também demandam avaliação futura mais sofisticada.

Para compreender motivos que possam estimulam a mobilização e adesão populacional a uma política sanitária, a partir da análise de redes sociais, houve um estudo de campo com 535 entrevistas residenciais em 4 diferentes bairros de Cedro investigando as conexões sociais dos moradores da cidade. Com isso, uma rede social contendo 1950 conexões interpessoais foi estruturada. A partir dela, nós (pessoas) centrais [Wasserman et al. 1994] foram selecionados. Os pontos nodais foram chamados analog 


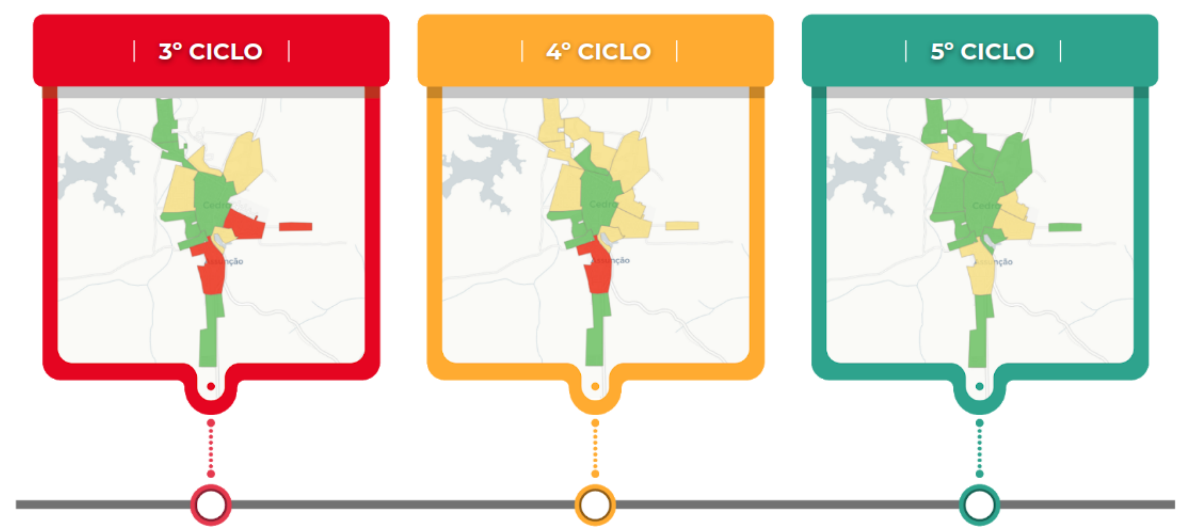

Figura 5. Ciclos de verificação com mutirões e a redução dos níveis de infestação.

influencers, visto que o estudo identificou estes vínculos partir de relações presenciais e independentes de cenários digitais. Com o esquema, idealizou-se uma estratégia de comunicação específica para tais influenciadores, a fim de torná-los os elementos centrais de utilização do aplicativo Aedes em Foco - Brigadas, em um processo similar ao de contágio [Berger 2020] em redes sociais online.

Como ação de comunicação foi realizada uma oficina de criação de lambe-lambes. Eles são utilizados como propaganda de baixo custo, fácil confecção e fazem uso de folhas de papel simples que são coladas em paredes e postes, contendo textos de fácil e rápida leitura. [Silva et al. 2015a] consideram que "essas produções têm natureza intercambiável entre a arte e a comunicação, pois apresentam características estilísticas de ambos os campos e agregam estratégias comunicativas. Essas estratégias buscam promover a articulação e a organização dessa mídia e o público."

Foram expostos exemplos e técnicas de produção e fixação. Logo após, foi solicitado aos ACEs que criassem, individualmente, frases relacionadas ao combate às arboviroses, fazendo uso da linguagem memética [Horta 2015]. Em 15 minutos, foram concebidas dezenas de frases como "Cedrense arretado acaba com o mosquito danado" e "Vizinho, cuide do seu baldinho". Os participantes elegeram seus textos favoritos, utilizando a técnica dotmocracy ${ }^{4}$. Logo após a escolha das frases, elas foram imediatamente aplicadas em um layout previamente concebido. A seguir, os protótipos foram impressos em laserjet, usando papeis no formato A4 de diferentes cores. Após apresentados e aprovados pelos participantes, centenas de cartazes foram confecionados. No dia seguinte, as peças foram fixadas pelos ACS, ACEs e voluntários, durante uma ação de mutirão em regiões com maior incidência de mosquitos e nas áreas mais movimentadas da cidade, como apresentado na Figura 6. Com a ação, houve uma adequação discursiva inédita da causa à população local, bem como a representação particular da causa e integração dos agentes e profissionais de saúde como equipe.

\section{Trabalhos relacionados}

O combate a doenças endêmicas, como a dengue, é uma questão histórica em vários países, porém ainda atacada de forma inespecífica. Mas há excepcionalidades inspiradoras.

\footnotetext{
${ }^{4}$ http://www.dotmocracy.org
} 


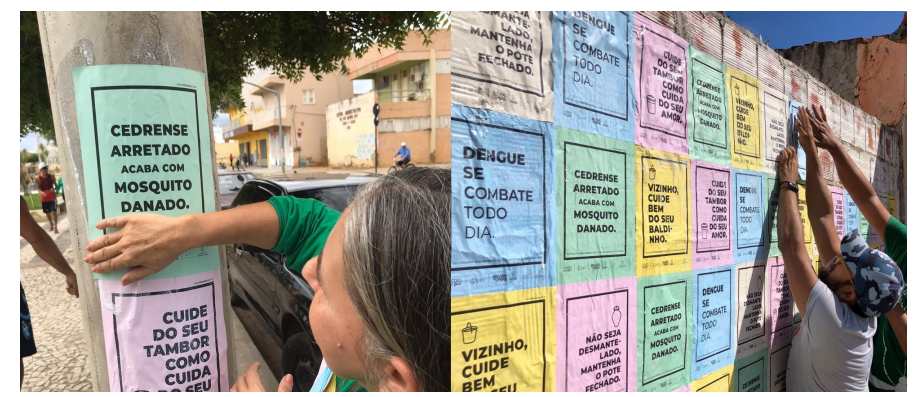

Figura 6. Colagem de lambe-lambes com linguagem memética - Cedro/CE.

O artigo [Lwin et al. 2019] apresenta um sistema de vigilância, comunicação e engajamento também baseado em elementos digitais, sistema este participante de um evento de hackatown ocorrido no Sri Lanka. [Rodriguez-Valero et al. 2018] registra uma plataforma de vigilância de sintomas compatíveis com arboviroses focada em participantes da delegação espanhola participante dos jogos olímpicos no Rio de Janeiro, dada a possibilidade de dispersão territorial dos vírus transmissores de arboviroses a partir de atletas infectados na cidade do evento. Já o trabalho [Fernandes Abel Mangueira et al. 2019] apresenta um quasi-experimento que trata do estudo comparativo das atitudes e conhecimento relacionados a arboviroses antes e após a aplicação de um curso online em smartphones. Os resultados apresentados demonstram empiricamente uma melhora de atitude em relação a prevenção de arboviroses após o curso.

Ciência cidadã é o foco de [Hamer et al. 2018], explorando a possibilidade do auxílio de cidadão, ajudando cientistas profissionais no processo de coleta de dados e de exemplares de artrópodes, que podem ser vetores de doenças endêmicas. Ele explora diversas fases similares às aplicadas aqui, como: educação e treinamento presenciais e criação de portais online e aplicativos para publicização dos dados obtidos. [Smolinski et al. 2017] traz um resumo de 23 ferramentas distintas de vigilância participativa, onde a população diretamente contribui com o monitoramento de doenças.

Infodengue [Codeco et al. 2018] é um sistema de monitoramento da situação epidemiológica que coleta de apresenta dados de diversas cidades brasileiras. Os dashboards apresentados neste sistema abrangem um bom número de municípios brasileiros porém não possuem riqueza de detalhes tal como proposta neste projeto. Tais trabalhos se relacionam a elementos do Aedes em Foco e, durante nossa pesquisa-ação, não foi encontrado exemplo que trabalhasse e integrasse todas as dimensões aqui propostas.

\section{Conclusão}

Este artigo apresentou o e-health Aedes em Foco para o combate a arboviroses. A aplicação da abordagem modificou a atuação de setores envolvidos com o combate a arboviroses na cidade de Cedro-CE. Com elementos baseados em software, educação presencial e on-line e uso da comunicação para a mobilização social, os resultados da pesquisa-ação mostram promissora viabilidade do Programa para transformar-se em instrumento basilar no combate a endemias, focadamente aquelas causadas pelo Aedes aegypti. O Programa demanda novas oportunidades de aplicação para seu aprimoramento, validação e escalabilidade, principalmente, nas ações e dispositivos direcionados à população e aplicação em grandes centros urbanos. Para tal, entendemos que a utilização de ideias baseadas em 
redes sociais para a mobilização social, mecanismos de bonificação à participação para a difusão de hábitos coletivos com o uso de tecnologias digitais apontam como promissoras proposições em uma direção divergente das tradicionais políticas públicas vinculadas à causa.

Agradecimentos: Agradecemos à Fundação Cearense de Apoio ao Desenvolvimento Científico e Tecnológico, Processo 5163154/2017, e à Prefeitura Municipal de Cedro-CE.

\section{Referências}

Araujo, I. S. d. (2012). As mídias, as instituições de saúde e a população: convergências e divergências na comunicação sobre a prevenção da dengue. Organicom, 9.

Berger, J. (2020). Contágio: por que as coisas pegam. Alta Books.

Brasil (2002). Programa nacional de controle da dengue. Fundação Nacional da Saúde. Ministério da Saúde, Brasília-DF.

Brasil (2009). Diretrizes nacionais para a prevenção e controle de epidemias de dengue. Secretaria de Vigilancia em Saúde. Ministério da Saúde, Brasília-DF.

Brasil (2012). Levantamento rápido de índices para aedes aegypti-liraa-para vigilância entomológica do aedes aegypti no brasil.

Bricks, L. F. (2004). Vacinas para a dengue: perspectivas. Pediatria, 26.

Cavalcante, C. E. (2013). Motivação no trabalho voluntário: delineamento de estudos no brasil. Estudos do CEPE.

Codeco, C. T. et al. (2018). Infodengue: A nowcasting system for the surveillance of arboviruses in brazil. Revue d'Épidémiologie et de Santé Publique, 66.

Coutinho, E. F., Junior, A. d. L. C., and Sarmento, W. W. F. (2013). Desenvolvimento de aplicações para educação à distância: $\mathrm{O}$ ambiente virtual de aprendizagem solar. In CBSOFT.

Duarte, H. H. P. and França, E. B. (2006). Qualidade dos dados da vigilância epidemiológica da dengue em belo horizonte, mg. Revista de Saúde Pública, 40.

Eysenbach, G. (2001). What is e-health? Journal of medical Internet research, 3.

Feitosa, F. R. S. et al. (2016). Indicadores de sustentabilidade como subsídio para a prevenção e controle da infestação pelo mosquito aedes aegypti no município de aracajuse.

Fernandes Abel Mangueira, F. et al. (2019). The prevention of arboviral diseases using mobile devices: a preliminary study of the attitudes and behaviour change produced by educational interventions. Tropical Medicine \& International Health.

Hamer, S. A., Curtis-Robles, R., and Hamer, G. L. (2018). Contributions of citizen scientists to arthropod vector data in the age of digital epidemiology. Current opinion in insect science, 28.

Horta, N. B. (2015). O meme como linguagem da internet: uma perspectiva semiótica. Brasília: Universidade de Brasília.

Junior, F. d. C. et al. (2013). Ciclos de vida comparados de aedes aegypti (diptera, culicidae) do semiárido da paraíba. Embrapa Algodão. 
Lwin, M. O. et al. (2019). Epihack sri lanka: development of a mobile surveillance tool for dengue fever. BMC medical informatics and decision making, 19.

McRoberts, M. (2015). Arduino Básico-2 ${ }^{a}$ edição: Tudo sobre o popular microcontrolador Arduino. Novatec Editora.

Neto, V. L. d. S. (2016). Elaboração e implementação de ferramenta educativa com ênfase na dengue, zika e chikungunya: relato de experiência. Revista Extendere, 4.

Nunes, M. d. O. et al. (2002). O agente comunitário de saúde: construção da identidade desse personagem híbrido e polifônico. Cadernos de Saúde Pública, 18.

Oliveira, A. E. C. d. et al. (2016). Implantação do e-SUS AB no Distrito Sanitário IV de João Pessoa (PB): relato de experiência. Saude em Debate, 40.

Oliveira, P. M. B., Júnior, E. O. C., and Cocco, D. D. A. (2017). A utilização do sal como larvicida no combate às larvas do aedes aegypti. Revista GeTeC, 6.

Pessoa, J. P. d. M., Oliveira, et al. (2016). Controle da dengue: os consensos produzidos por agentes de combate às endemias e agentes comunitários de saúde sobre as ações integradas. Ciência \& Saúde Coletiva, 21:2329-2338.

Pessoa, J. P. d. M., Oliveira, E. S. F. d., Lemos, C. S., and Teixeira, R. A. G. (2013). Integração e articulação intersetorial no controle da dengue: a percepção dos agentes de combate de endemias. Indagatio Didactica, 5.

Quintão, M. S. (2016). X-dengue: game design e estratégias de gamificação de um jogo educativo sobre a dengue.

Rodriguez-Valero, N. et al. (2018). Mobile based surveillance platform for detecting zika virus among spanish delegates attending the rio de janeiro olympic games. PloS one, 13.

Rosa, A. P. d. A. T. et al. (2000). Arboviroses.

Santos, A. et al. (2018). Seleção do Método de Pesquisa: Guia para pós graduando em design e áreas afins. Insight.

Schall, V. T. et al. (2015). Educação em saúde como estratégia no controle integrado da dengue: reflexões e perspectivas. Fundação Oswaldo Cruz.

Silva, H. T. et al. (2015a). Desvios: Cartaz lambe-lambe, comunicação visual e arte nos espaços de trânsito.

Silva, I. B. d., Mallmann, D. G., and de Vasconcelos, E. M. R. (2015b). Estratégias de combate à dengue através da educação em saúde: uma revisão integrativa. Saúde (Santa Maria), 41.

Smolinski, M. S. et al. (2017). Participatory disease surveillance: engaging communities directly in reporting, monitoring, and responding to health threats. JMIR public health and surveillance, 3 .

Valle, D., Pimenta, D. N., and Aguiar, R. (2016). Zika, dengue e chikungunya: desafios e questões. Epidemiologia e Serviços de Saúde, 25.

Wasserman, S., Faust, K., et al. (1994). Social network analysis: Methods and applications, volume 8 . Cambridge university press. 